\title{
Dramatic efficacy of dabrafenib in Erdheim-Chester disease (ECD): a pediatric patient with multiple large intracranial ECD lesions hidden by refractory Langerhans cell histiocytosis
}

\author{
${ }^{*}$ Xiaolei Hao, MD, ${ }^{1}$ Ruie Feng, MD, ${ }^{2}$ Yalan Bi, MD, ${ }^{2}$ Yuhan Liu, MD, ${ }^{1}$ Chunde Li, MD, ${ }^{1}$ Tao Lu, MS, ${ }^{2}$ \\ and Yongji Tian, MD' \\ 1Department of Pediatric Neurosurgery, Beijing Tiantan Hospital, Capital Medical University, China National Clinical Research \\ Center for Neurological Diseases, Center of Brain Tumor, Beijing Institute for Brain Disorders, Beijing Key Laboratory of Brain \\ Tumor; and 'Department of Pathology, Peking Union Medical College Hospital, Chinese Academy of Medical Sciences and \\ Peking Union Medical College, Beijing, People's Republic of China
}

\begin{abstract}
Erdheim-Chester disease (ECD) is a rare non-Langerhans cell form of histiocytosis that can affect the central nervous system. ECD predominantly affects adults, and only a few pediatric cases have been reported. The co-occurrence of ECD and Langerhans cell histiocytosis $(\mathrm{LCH})$ is exceedingly rare. An 11-year-old boy, who was diagnosed with LCH 7 years previously, presented with multiple giant intracranial lesions. At the time of his initial diagnosis, only one intracranial lesion was observed, and it began to enlarge. Currently, up to 7 intracranial lesions can be observed in this patient. However, the diagnosis of ECD was not confirmed until this most recent open resection. The BRAF V600E mutation was detected in both LCH and ECD lesions. Dabrafenib therapy exhibited dramatic efficacy in this pediatric patient. This case represents the first successful application of dabrafenib in a pediatric patient with intracranial ECD lesions as well as mixed ECD and $\mathrm{LCH}$. In this article, the authors describe the intricate diagnosis and treatment processes in this patient. Recent studies regarding treatment with $B R A F$ inhibitors for neurological involvement in mixed ECD and LCH are also reviewed.
\end{abstract}

https://thejns.org/doi/abs/10.3171/2018.6.PEDS17728

KEYWORDS Erdheim-Chester disease; Langerhans cell histiocytosis; dabrafenib; brain tumor; oncology; treatment

$\mathrm{E}$ RDHEIM-Chester disease (ECD) is a rare nonLangerhans cell form of histiocytosis. Currently, no more than 750 cases have been reported. ECD predominantly affects adults, but rare pediatric cases have been reported, with fewer than 20 known cases to date. 7 Historically, ECD typically manifests as sclerotic lesions in the bone (95\% of patients), but many diverse organ systems can be involved, including the central nervous system (25\%-50\% of patients). ${ }^{3}$

Langerhans cell histiocytosis (LCH) and ECD have similar clinical presentations, such as diabetes insipidus and exophthalmos. In contrast, the incidence of $\mathrm{LCH}$ in adults is significantly lower than it is in children. LCH most frequently affects the bone (80\% of patients), but LCH lesions are osteolytic and are usually localized to the skull. ${ }^{10}$

In recent studies, $B R A F$ inhibitors have shown a significant impact on the management of these diseases. However, data regarding the usage and efficacy of $B R A F$ inhibitors in pediatric ECD and $\mathrm{LCH}$ are lacking. In this article, we report a unique case of a pediatric patient with $\mathrm{LCH}$ and multiple intracranial ECD lesions. These large intracranial ECD lesions have not been previously reported in children. In this rare case, the $B R A F$ V600E mutation was detected in both LCH and ECD lesions. Moreover, dabrafenib therapy exhibited dramatic efficacy in this pediatric patient.

ABBREVIATIONS ECD = Erdheim-Chester disease; $\mathrm{FGD}={ }^{18} \mathrm{~F}$-labeled fluorodeoxyglucose; $\mathrm{LCH}=$ Langerhans cell histiocytosis.

SUBMITTED December 21, 2017. ACCEPTED June 20, 2018.

INCLUDE WHEN CITING Published online September 28, 2018; DOI: 10.3171/2018.6.PEDS17728.

* X.H. and R.F. contributed equally to this work and share first authorship. 


\section{Case Report}

History and Examination

An 11-year-old boy was admitted to the regional hospital in September 2009 for exophthalmos and generalized bone pain. A 3D CT scan of the skull showed osteolytic lesions in the craniofacial bones (Fig. 1A). An MRI scan of the brain revealed a thickened pituitary stalk, a lack of hyperintense signal in the neurohypophysis, and a nodule located in the trigone of the left lateral ventricle (Fig. $1 \mathrm{~B}$ and $\mathrm{C}$ ). A diagnostic open biopsy was performed on a right frontal bone lesion, and microscopic examination indicated $\mathrm{LCH}$. Over the next 5 years, the patient received chemotherapy based on the appropriate arm of the LCHI, LCH-II, and LCH-III protocols. The drugs used in the regimens included prednisone, vincristine, etoposide, and mercaptopurine. The exophthalmos was relieved, and the number of bone lesions gradually decreased, but the intracranial lesions continued to grow in size. In January 2011, the patient underwent resection of the enlarged nodule (Fig. 1D) located in the trigone of the left lateral ventricle; the tumorous lesion was completely resected. The surgical specimens showed a histiocytic appearance with a mixture of foamy histiocytes and a fibrotic stromal background. Immunostaining revealed tissue positive for CD68 and negative for CD1a and S100. A diagnosis of xanthogranuloma was suggested. Chemotherapy was terminated in the last 2 years because his parents thought that the patient's condition had improved, and they were worried about the side effects of the chemotherapeutic drugs. The patient's condition was relatively stable over a period of time. Later, the exophthalmos worsened, and the patient gradually lost vision. He was then referred to our hospital.

The patient presented with intermittent headache and generalized bone pain. On repeated contrast-enhanced brain MRI, tumorous lesions showed homogeneous enhancement (no local recurrence in the trigone of the left lateral ventricle [Fig. 2A]). Diffuse bone destruction was observed in the 3D skull. Radiographic results demonstrated patchy and increased nodular density in the right tibiofibular and foot bones (Fig. 3A). On examination, he presented with a developmental delay. Both eyes bulged severely, and multiple scalp masses were palpable. Xanthogranuloma is a pathological change in some histiocytoses, such as juvenile xanthogranuloma and adult xanthogranuloma. Moreover, osteosclerosis is not characteristic of LCH and these diseases. Thus, further investigation should be performed. Hypoplasia and headache were attributed to the significant occupying effect of the multiple large intracranial lesions. Considering the risk of surgery for the saddlearea lesion, his parents refused. To obtain another biopsy specimen and reduce intracranial pressure, we performed an operation for integral resection of another larger tumorous lesion, which was located in the right parietal lobe.

\section{Operation and Histopathological Findings}

We made a horseshoe-shaped skin incision and parietal craniotomy on the right side. During the operation, we
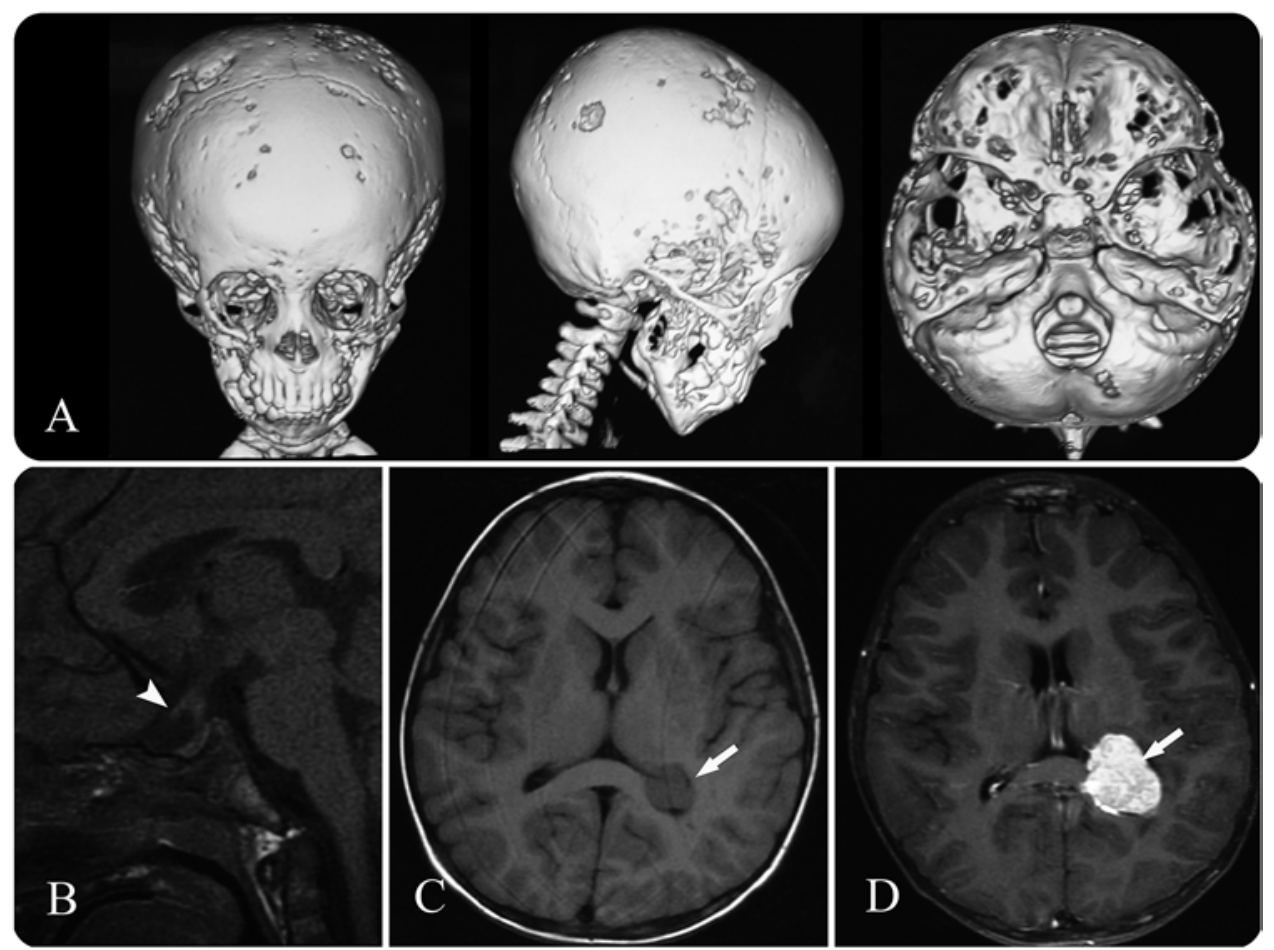

FIG. 1. A: Three-dimensional CT scans of the skull showing osteolytic lesions in the craniofacial bones. B and C: Sagittal and axial T1-weighted MR images obtained 7 years ago. Images show a thickening pituitary stalk, lack of hyperintense signal in the neurohypophysis ( $\mathrm{B}$, arrowhead), and a nodular mass with isointense signals located in the trigone of the left lateral ventricle $(C, 1.5 \times 1.5 \times 1.5 \mathrm{~cm}$, arrow). D: Axial T1-weighted Gd-enhanced MR image acquired after the initial chemotherapy. The image shows that the nodular mass with homogeneous intense enhancement is larger now (arrow, maximal diameter $3.0 \mathrm{~cm}$ ). 

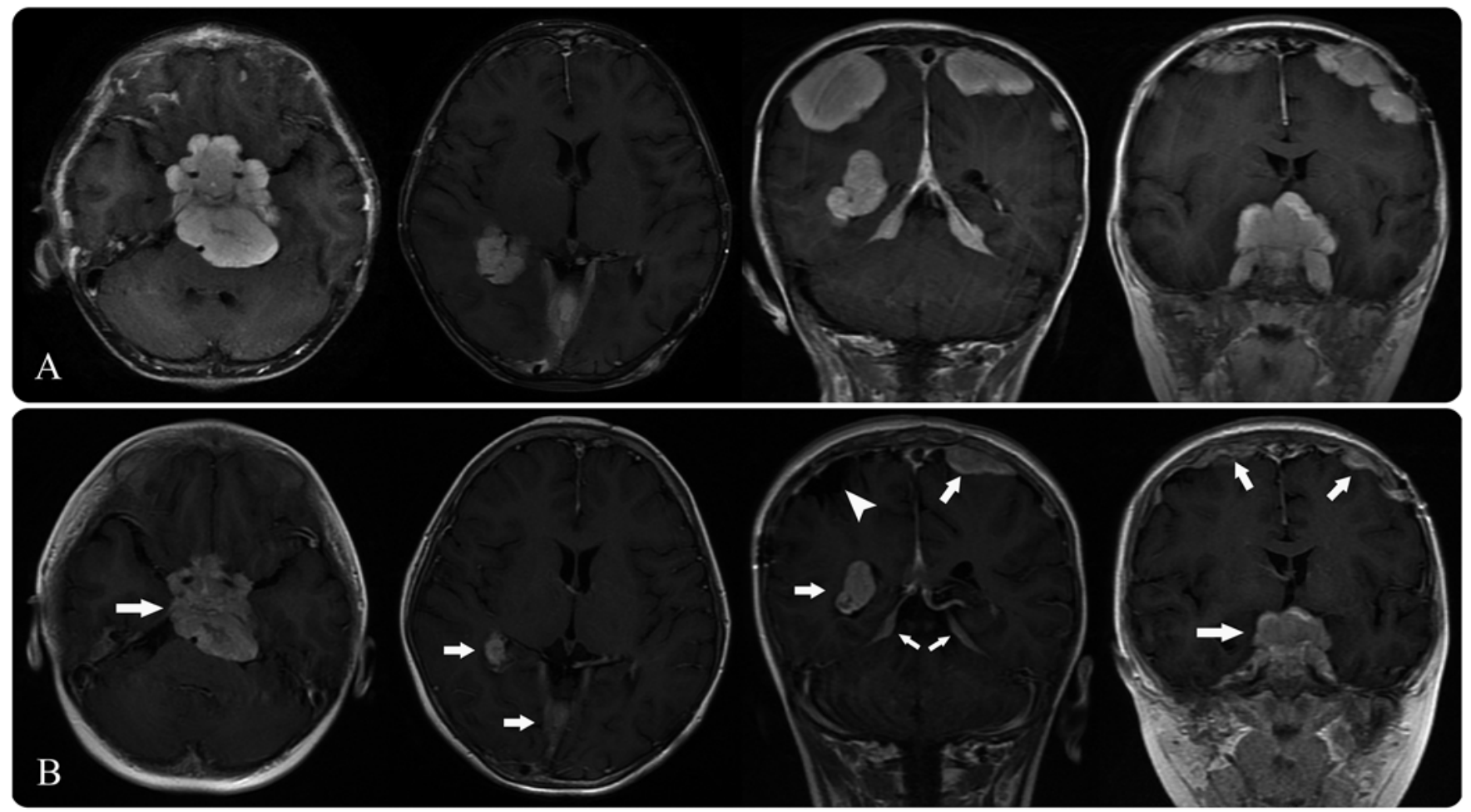

FIG. 2. A: Axial and coronal T1-weighted Gd-enhanced MR images obtained before the recent operation. The images show the mass lesions involving the saddle area $(5.8 \times 4.7 \times 4.5 \mathrm{~cm})$, right lateral ventricle, bilateral frontal and parietal lobes, cerebral falx, and tentorium cerebelli. B: Follow-up MR images acquired after 2 months of dabrafenib therapy. The lesion located in the right parietal lobe was resected completely in the recent operation (arrowhead). The other intracranial mass lesions showed a marked decrease in size (arrows).

found a yellow solid mass with a clear boundary located in the convexity of the parietal lobe. The lesion had a normal blood supply and invaded the dura. The lesion was integrally resected.

The tumorous lesion was approximately $5.5 \times 5.0 \times 5.0$ $\mathrm{cm}$ with a solid yellow cut surface (Fig. 4A). The lesion tissue revealed infiltration of foamy mononucleated histiocytes with background fibrosis (Fig. 4B). Immunohistochemical staining revealed that the histiocytes were negative for CD1a and langerin (CD207) and positive for CD68 (Fig. 4C). These features were the same as the previous biopsy specimen obtained 6 years prior. The first specimen from the right frontal bone lesion was also reviewed to confirm the LCH diagnosis. Immunohistochemical staining revealed the histiocytes positive for CD1a and S100, which are markers of Langerhans cells (Fig. 4D-F).

\section{Molecular Studies}

Formalin-fixed, paraffin-embedded tissue samples were used to extract DNA from the tissue lesions. Through pyrosequencing, the LCH lesion and the recent specimen obtained from surgery were confirmed as positive for the $B R A F$ V600E mutation. The BRAF V600E mutation was also detected in cell-free DNA extracted from the peripheral blood.

\section{Pathological Diagnosis and Postoperative Course}

Based on the clinical features, histomorphological fea-
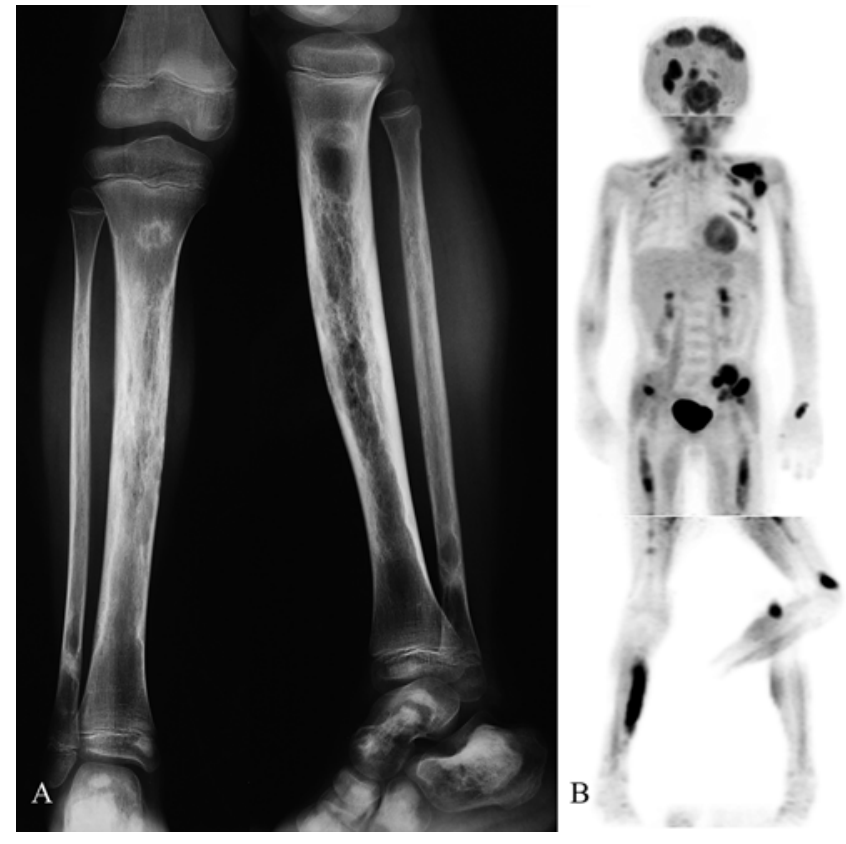

FIG. 3. A: Radiograph of the right tibiofibular and foot bones demonstrating the coexistence of osteolytic and sclerotic changes. B: FDGPET scan revealing bilateral radiotracer uptake in the ribs, humeri, ilium, and femurs. 


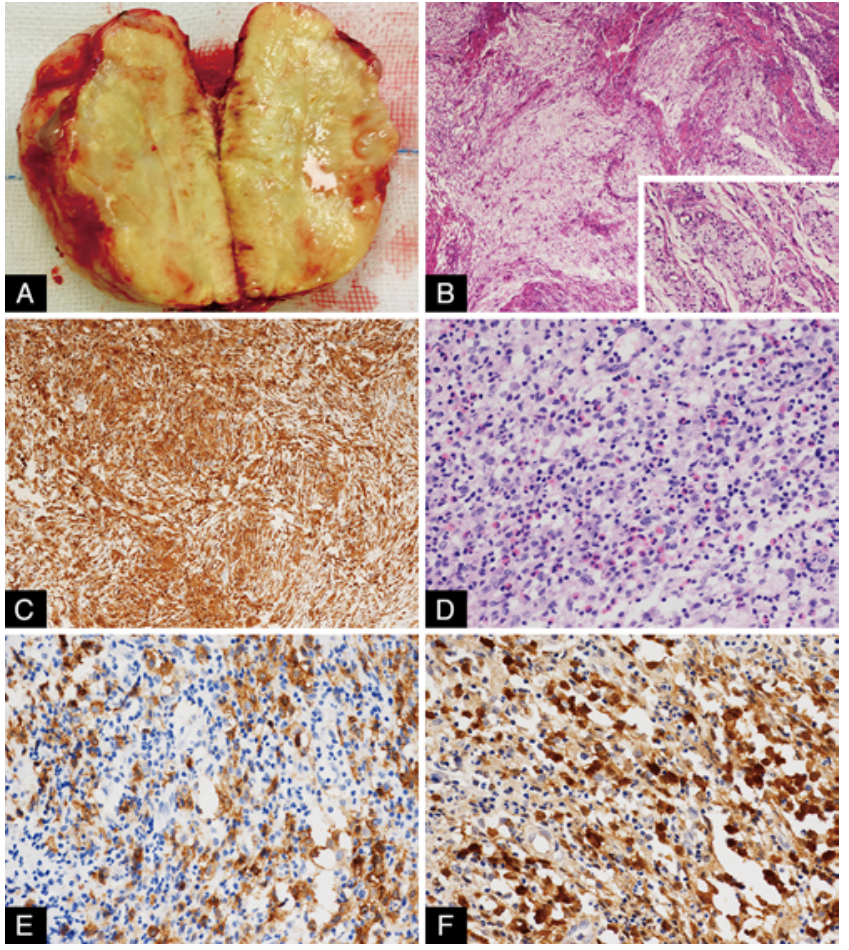

FIG. 4. Photographic and histological studies. A: The ECD lesion resected from the convexity of the right parietal lobe, which has a yellow and solid cut surface. B: The ECD specimen shows foamy histiocytes in a background of fibrosis. $\mathrm{H} \& \mathrm{E}$, original magnification $\times 200$. Highermagnification photomicrograph reveals that the foamy histiocytes have bland nuclei and abundant cytoplasm. $\mathrm{H} \& \mathrm{E}$, original magnification $\times 400$ (inset). C: Immunohistochemical staining shows many of the CD68-immunopositive foamy cells in the ECD specimen. CD68, original magnification $\times 400$. D: The LCH specimen shows Langerhans cells that have coffee bean-shaped nuclei. H \& E, original magnification $\times 200$. E and F: These cells reacted positively to CD1a (E) and S100 (F). Figure is available in color online only.

tures, and immunophenotype, the two intracranial lesions were diagnosed as ECD. A diagnosis of mixed ECD and LCH for this patient was established (Table 1).

The patient's headache subsided after surgery. The chemotherapeutic regimen was changed to cladribine and cytarabine. This regimen dramatically improves the survival of patients with a risk of organ involvement. Cladribine also has been used in the treatment of both newly diagnosed and refractory ECD. However, after 5 days of treatment, serious pancytopenia occurred. PET with ${ }^{18} \mathrm{~F}-$ labeled fluorodeoxyglucose (FDG) of the entire body was performed to evaluate systemic involvement (Fig. 3B). His parents then requested another regimen because of the side effects and high cost of the current chemotherapeutic regimen. Considering that the patient harbored the $B R A F$ V600E mutation, this regimen was stopped, and $B R A F$ inhibitor dabrafenib therapy was started at a dose of $100 \mathrm{mg}$ once daily. After 1 week, the patient complained of thigh muscle pain, and the dose was reduced to $50 \mathrm{mg}$ once daily. Following several days of treatment, the exophthalmos remarkably decreased, the mandibular pain resolved, and the scalp masses disappeared. After 2 months of dabrafenib therapy, a head MRI scan showed a marked decrease in the size of the intracranial tumorous lesions (Fig. 2B). After the 4th month, repeat brain MRI showed no significant change in the size of the intracranial tumorous lesions. Currently, the patient is in a stable condition. His general status is excellent without any side effects, and no new lesions have appeared. This response allowed us to delay surgery for the saddle-area lesion.

\section{Discussion}

The pathogenesis of $\mathrm{LCH}$ and ECD remains speculative. Whether ECD and $\mathrm{LCH}$ are inflammatory disorders that are manifestations of an immunological aberration or neoplasms remains to be determined. ${ }^{7}$ Recent studies have confirmed that $50 \%-60 \%$ of LCH samples have BRAF V600E mutations. ${ }^{1}$ Similarly, $50 \%-60 \%$ of cases with ECD are positive for $B R A F$ V600E. ${ }^{6}$ The parallel prevalence of the BRAF V600E mutation in ECD and LCH indicates a common origin cell for both proliferations. This evidence supports the hypothesis that $\mathrm{LCH}$ and ECD are neoplastic in nature. Based on the similar clinical complications of LCH and ECD, the Histiocyte Society recommended inclusion of both conditions in the "L" (Langerhans) group..$^{5}$

Mixed ECD and LCH (i.e., coexisting ECD and LCH) is exceptionally rare and included in the "L" group. ${ }^{5}$ In general, LCH and ECD can be distinguished by histological examination. However, $20 \%$ of patients with ECD also have LCH lesions, sometimes within the same lesion tissues. ${ }^{8}$ Since the diagnosis of ECD never precedes LCH, and both disorders have similar clinical complications, ${ }^{8}$ ECD can be easily overlooked in LCH patients.

In this patient, ECD was neglected at first because of the extreme rarity of mixed ECD and LCH in children. The poor response of the intracranial ECD lesions to chemotherapy allowed the tumorous lesions to continue growing. Surgical treatment for patients who have intracranial tumorous lesions is not common. Whether recurrence of lesions in situ will occur after integral resection has not been reported. In addition, for this patient, there is

TABLE 1. Summary of the 3 biopsy results

\begin{tabular}{cllll}
\hline Biopsy Samples & Op Date & \multicolumn{1}{c}{ Lesion Site } & Immunophenotype & Pathological Diagnosis \\
\hline 1st & 7 yrs ago & Rt frontal bone & CD1a pos, S100 pos & LCH $^{*}$ \\
\hline 2nd & 6 yrs ago & Trigone of It lat ventricle & CD1a neg, CD68 pos & 1st: xanthogranuloma; current: ECD \\
\hline 3rd & Recent op & Rt parietal lobe & CD1a neg, CD68 pos & ECD* $^{*}$ \\
\hline
\end{tabular}

Neg = negative; pos = positive .

* BRAF V600E mutation detected using real-time polymerase chain reaction. 
a high chance that endocrine function and vision will be improved by resection of the saddle-area lesion. However, resection of the other larger tumorous lesions to reduce intracranial pressure had a considerable effect.

The breakthrough for the therapeutic regimens was the use of $B R A F$ inhibitors based on the discovery of $B R A F$ mutations in ECD and LCH. ${ }^{1,6}$ Currently, regimens including $B R A F$ inhibitors are under investigation. In the Cohen Aubart et al. and Diamond et al. studies, vemurafenib demonstrated clinically meaningful, long-term efficacy in adult patients with $B R A F$ V600E mutant-isolated ECD and -isolated $\mathrm{LCH}^{2,4}$ In previous mixed $\mathrm{ECD}$ and $\mathrm{LCH}$ cases, $B R A F$ V600E-positive LCH lesions sometimes occurred concomitant with $B R A F$ V600E-positive ECD lesions. ${ }^{8}$ In this condition, the effect of $B R A F$ inhibitors has not been identified, especially for children. Treatment with vemurafenib against ECD lesions has been reported only in a single case-a 2-year-old patient with $B R A F$-positive LCH and ECD. ${ }^{12}$ The optimal dose of and duration of treatment with vemurafenib and dabrafenib for ECD and $\mathrm{LCH}$ and mixed ECD and $\mathrm{LCH}$ have not been identified. However, for ECD and $\mathrm{LCH}$, doses below the currently indicated dose for melanoma may be sufficient to maintain therapeutic responses. ${ }^{4}$ Most patients experience a recurrence of the tumor following interruption of $B R A F$ inhibitor treatment. ${ }^{2}$ In the case of our patient, using a lower-dose dabrafenib, responses were observed at all disease sites, especially for the intracranial ECD lesions and osteolytic LCH lesions in the skull. This result suggests that a lower-dose dabrafenib is also effective for pediatric patients with mixed ECD and LCH. Treatment resistance during BRAF inhibition may occur in ECD and $\mathrm{LCH}$, as seen in other $B R A F$-mutant cancers. Similarly, our patient initially showed a marked response after initiation of $B R A F$ inhibition, but he gradually developed a resistance to this therapy. Nordmann et al. published a case report of a patient with ECD in whom treatment resistance developed as a result of a secondary MAPK pathway-activating mutation. ${ }^{11}$ Our patient's condition is currently stable, but dabrafenib should not be stopped to avoid a relapse. New mutational analysis of the treatment-resistant ECD lesions should be performed after obtaining consent from his parents.

According to our review of the literature, two children with mixed ECD and LCH have been reported., ${ }^{9,12}$ The intracranial lesions in our case are more expansile and voluminous than those of the two previously reported cases. Such large lesions in the sellar region have also not been reported in patients with ECD. Therefore, it was necessary to completely resect the large tumorous lesion before initiating adjuvant chemotherapy to reduce intracranial pressure. This new specimen also helped us to establish the diagnosis of ECD. The detection of the BRAF V600E mutation in the current ECD lesion and the previous LCH lesion allowed us to treat the patient with dabrafenib. Fortunately, marked efficacy has been observed. This case is the first involving the successful application of dabrafenib in a pediatric patient with intracranial ECD lesions as well as mixed ECD and LCH. The clinical course of our patient involved the establishment of early, accurate diagnoses and controlling intracranial tumorous lesion devel- opment through rational chemotherapy, which is very important for mixed ECD and LCH patients. Furthermore, this is the first report of a patient with 6 years of followup and no local recurrence after undergoing intracranial ECD lesion integral resection. We suggest that dabrafenib should be considered as an off-label therapy in pediatric patients with mixed ECD and LCH. In addition, early surgical treatment should be considered for patients in whom response to chemotherapy and targeted therapy is absent.

\section{Acknowledgments}

Dr. Tian was partially supported by the Beijing Municipal Administration of Hospitals' Youth Program (QML20150501) and Beijing Natural Science Foundation (7172041). Dr. Bi was partially supported by the Beijing Key Laboratory of Head and Neck Molecular Diagnostic Pathology (2016TJBF01).

\section{References}

1. Badalian-Very G, Vergilio JA, Degar BA, MacConaill LE, Brandner B, Calicchio ML, et al: Recurrent BRAF mutations in Langerhans cell histiocytosis. Blood 116:1919-1923, 2010

2. Cohen Aubart F, Emile JF, Carrat F, Charlotte F, Benameur $\mathrm{N}$, Donadieu J, et al: Targeted therapies in 54 patients with Erdheim-Chester disease, including follow-up after interruption (the LOVE study). Blood 130:1377-1380, 2017

3. Diamond EL, Dagna L, Hyman DM, Cavalli G, Janku F, Estrada-Veras J, et al: Consensus guidelines for the diagnosis and clinical management of Erdheim-Chester disease. Blood 124:483-492, 2014

4. Diamond EL, Subbiah V, Lockhart AC, Blay JY, Puzanov I, Chau I, et al: Vemurafenib for BRAF V600-mutant ErdheimChester Disease and Langerhans cell histiocytosis: analysis of data from the histology-independent, phase 2, open-label VE-BASKET study. JAMA Oncol 4:384-388, 2018

5. Emile JF, Abla O, Fraitag S, Horne A, Haroche J, Donadieu $\mathrm{J}$, et al: Revised classification of histiocytoses and neoplasms of the macrophage-dendritic cell lineages. Blood 127:26722681, 2016

6. Haroche J, Charlotte F, Arnaud L, von Deimling A, HéliasRodzewicz Z, Hervier B, et al: High prevalence of BRAF V600E mutations in Erdheim-Chester disease but not in other non-Langerhans cell histiocytoses. Blood 120:27002703, 2012

7. Haroche J, Cohen-Aubart F, Rollins BJ, Donadieu J, Charlotte F, Idbaih A, et al: Histiocytoses: emerging neoplasia behind inflammation. Lancet Oncol 18:e113-e125, 2017

8. Hervier B, Haroche J, Arnaud L, Charlotte F, Donadieu J, Néel A, et al: Association of both Langerhans cell histiocytosis and Erdheim-Chester disease linked to the BRAFV600E mutation. Blood 124:1119-1126, 2014

9. Kim S, Lee M, Shin HJ, Lee J, Suh YL: Coexistence of intracranial Langerhans cell histiocytosis and ErdheimChester disease in a pediatric patient: a case report. Childs Nerv Syst 32:893-896, 2016

10. Laurencikas E, Gavhed D, Stålemark H, van’t Hooft I, Prayer $\mathrm{D}$, Grois N, et al: Incidence and pattern of radiological central nervous system Langerhans cell histiocytosis in children: a population based study. Pediatr Blood Cancer 56:250-257, 2011

11. Nordmann TM, Juengling FD, Recher M, Berger CT, Kalbermatten D, Wicki A, et al: Trametinib after disease reactivation under dabrafenib in Erdheim-Chester disease with both $B R A F$ and $K R A S$ mutations. Blood 129:879-882, 2017 
12. Váradi Z, Bánusz R, Csomor J, Kállay K, Varga E, Kertész $\mathrm{G}$, et al: Effective BRAF inhibitor vemurafenib therapy in a 2-year-old patient with sequentially diagnosed Langerhans cell histiocytosis and Erdheim-Chester disease. OncoTargets Ther 10:521-526, 2017

\section{Disclosures}

The authors report no conflict of interest concerning the materials or methods used in this study or the findings specified in this paper.

\section{Author Contributions}

Conception and design: Tian, Hao, Feng. Acquisition of data: Hao. Analysis and interpretation of data: $\mathrm{Hao}, \mathrm{Bi}, \mathrm{Liu}, \mathrm{Li}, \mathrm{Lu}$. Drafting the article: Hao. Critically revising the article: Tian, Feng. Reviewed submitted version of manuscript: Tian, Feng, Bi. Approved the final version of the manuscript on behalf of all authors: Tian.

\section{Correspondence}

Yongji Tian: Capital Medical University, Beijing, People's Republic of China. email: ttyysw1tyj@163.com. 